FORMATION Formation emploi

Revue française de sciences sociales

133 | Janvier-Mars 2016

Heurs et malheurs de l'apprentissage en Suisse

\title{
Entre trouver et se trouver une place d'apprentissage : quand la différence se joue dans la personnalisation du processus
}

Getting an apprenticeship: When the importance to individualize the application process makes a significant difference

Die Individualisierung des Bewerbungsprozesses als Erfolgsrezept auf der Suche nach einem Ausbildungsplatz

Entre encontrar y encontrarse un lugar de aprendizaje : cuando la diferencia se juega en la personalización del proceso

Guillaume Ruiz et Gaële Goastellec

(2) OpenEdition

Journals

Édition électronique

URL : http://journals.openedition.org/formationemploi/4663

DOI : $10.4000 /$ formationemploi.4663

ISSN : 2107-0946

Éditeur

La Documentation française

Édition imprimée

Date de publication : 20 avril 2016

Pagination : 121-138

ISSN : 0759-6340

Référence électronique

Guillaume Ruiz et Gaële Goastellec, «Entre trouver et se trouver une place d'apprentissage : quand la différence se joue dans la personnalisation du processus », Formation emploi [En ligne], 133 | JanvierMars 2016, mis en ligne le 21 avril 2018, consulté le 30 octobre 2020. URL : http:// journals.openedition.org/formationemploi/4663; DOI : https://doi.org/10.4000/formationemploi.4663

(c) Tous droits réservés 


\section{Entre trouver et se trouver une place d'apprentissage : quand la différence se joue dans la personnalisation du processus}

Guillaume Ruiz

Assistant-doctorant en sciences sociales à l'université de Lausanne. Sa thèse en sociologie de l'éducation porte sur le rapport au temps des jeunes en formation professionnelle initiale dans

le canton de Vaud en Suisse

GAËLE GOASTELLEC

Sociologue, maître d'enseignement et de recherche à l'université de Lausanne. Ses recherches principales portent sur la comparaison socio-historique des systèmes d'enseignement supérieur et plus largement sur la question des inégalités sociales dans les trajectoires individuelles et les

politiques scolaires

Résumé

Entre trouver et se trouver une place d'apprentissage : quand la différence se joue dans la personnalisation du processus

En Suisse, les deux tiers des jeunes passent, après la scolarité obligatoire, par la formation professionnelle initiale. Si ce modèle de formation est prisé dans le panorama international, les processus par lesquels s'obtient une place d'apprentissage demeurent mal connus. A partir d'une enquête qualitative auprès d'apprentis et de recruteurs, cet article dévoile le " curriculum caché » dans la recherche d'une place d'apprentissage et ses conséquences pour les candidats à l'apprentissage. Ce curriculum comprend la maîtrise de certains savoir-être, variables selon le type de monde en vigueur au sein des différentes entreprises.

Mots clés : enseignement technique-professionnel, apprentissage, recrutement, critère d'embauche, Suisse

Abstract

Getting an apprenticeship: When the importance to individualize the application process makes a significant difference

In Switzerland, the basic vocational training system is attended by two thirds of the students finishing compulsory schooling. If this training model seems an appropriate remedy in the contemporary international landscape, little is known about the processes by which an apprenticeship is obtained. On the basis of a qualitative survey of apprentices and recruiters, this article unveils the " hidden curriculum " in the seeking of an apprentice- 
ship position and its consequences for the students. This curriculum includes the mastery of a certain know-how depending of the type of world at play in the different companies.

Keywords : technical \& vocational education, apprenticeship, recruitment, hiring criteria, Switzerland

Journal of Economic Literature: M 53, M 51

Traduction : Auteur.e

Comparée à l'Union européenne, qui affichait un taux de chômage de $23.3 \%$ chez les jeunes actifs de moins de 25 ans en 2013 (Eurostat, 2014), la Suisse faisait, avec $7 \%$ chez les 15-24 ans (OFS, 2014), plutôt figure de bonne élève.

Bien que deux fois plus élevé que pour l'ensemble de la population active du pays, ce faible taux de chômage et sa relative stabilité dans le temps ont récemment suscité un important regain d'intérêt, sur la scène internationale, pour le système de formation professionnelle helvétique.

La Commission européenne y voit notamment un instrument de lutte contre le chômage des jeunes (Commission européenne, 2010). En Suisse, et malgré les disparités entre cantons, le système de formation professionnelle initiale, communément nommé "l'apprentissage ", est fréquenté par les deux tiers des jeunes à la sortie de leur scolarité obligatoire (SEFRI, 2014). Parmi eux, 80 \% optent pour l'une des 250 formations duales (SEFRI, 2014), soit en alternance entre une formation pratique au sein d'une entreprise formatrice et des cours théoriques en école.

Ce modèle de formation apparait donc comme une solution idéale dans le panorama international ; pour autant, les recherches menées depuis le début des années 2000 ont révélé l'existence de mécanismes ségrégatifs lors de la recherche d'une place, que ce soit à l'entrée de l'apprentissage (Imdorf \& Seiterle, 2014) ou à la sortie, lorsqu'il s'agit de s'insérer comme professionnel sur le marché de l'emploi (Fibbi et al., 2003).

D'autres recherches ont étudié les facteurs de réussite durant la formation (Häfeli \& Schellenberg, 2009), mis en avant les difficultés rencontrées par les apprentis dans leur construction identitaire sexuée et professionnelle (Lamamra, 2014) et étudié les raisons motivant certains à abandonner leur formation (Lamamra \& Masdonati, 2009).

L'étude TREE (Transition de l'Ecole à l'Emploi), basée sur un suivi longitudinal d'une cohorte d'élèves sortis de l'école en 2000, a apporté un éclairage structurel, soulignant la non-linéarité des parcours et le poids des solutions transitoires entre l'école et l'apprentissage (Amos, 2007).

Mais cet espace de formation demeure trop peu étudié en regard de la place qu'il occupe au sein du système de formation suisse (Cortesi \& Imdorf, 2013). En particulier, les pro- 
cessus par lesquels se construit l'accès à une place d'apprentissage et qui déterminent la possibilité de suivre la formation, demeurent mal connus.

Comme le relève Amos (2007), bien que le système de formation ait été pensé pour permettre une transition directe entre la fin de l'école obligatoire et le secondaire II - soit l'entrée en formation professionnelle initiale, en école de culture générale, ou en gymnase (équivalent du lycée) - dans les faits, nombre de parcours ne sont pas linéaires.

La crise conjoncturelle et l'augmentation du chômage qui a frappé la Suisse dans les années 90, provocant un important déséquilibre sur le marché de l'apprentissage notamment en raison des restrictions adoptées par les entreprises dans l'engagement d'apprentis (Valli, 2012), ont accentué ce phénomène.

Dans ce contexte ont émergé des mesures transitoires pour accueillir les jeunes (15-25 ans) sans solution de formation à la sortie de l'école obligatoire, souvent faute d'avoir trouvé une place d'apprentissage. Couvrant une période de six mois à une année, ces structures d'aide à la transition ${ }^{1}$ proposent des activités pratiques et scolaires, des stages en entreprise, du conseil en orientation et de l'aide à la recherche d'une place d'apprentissage (DFJC, 2014). Elles accueillent aujourd'hui environ 20 \% d'une classe d'âge dans le canton de Vaud (SCRIS, 2011).

Pour autant, depuis quelques années, une partie des places offertes demeurent vacantes (SEFRI 2013), notamment parce que certains dossiers de candidatures sont jugés inappropriés par les entreprises formatrices (LINK, 2013) mais aussi parce que certains secteurs, comme l'hôtellerie et le bâtiment, sont désertés par les jeunes. Comme le relève Sigerist (2003, p. 21) : "choisir un apprentissage dans ces secteurs ne semble pas offrir aux jeunes un futur réel, d'autant plus que les possibilités de changer de profession et de pouvoir transférer ses connaissances d'un CFC [Certificat fédéral de capacité] à un autre paraissent aléatoires ». Certains emplois, voire certains secteurs, apparaissent également boudés par les futurs apprentis en raison de la faible valorisation sociale qu' ils apporteraient.

A l'inverse, certains apprentissages, comme celui d'employé de commerce, sont prisés par les jeunes (SEFRI, 2014). Il en va de même des apprentissages en lien avec l'informatique, l'industrie de transformation, la vente ou encore la santé et le social. Cette demande concentrée sur certaines formations contribue à créer une concurrence importante et à accentuer certaines formes de discrimination à l'égard de certains groupes issus de l'immigration (Imdorf \& Seiterle, 2015) ou liées au genre (Lamamra, 2011). Cette concurrence est également stimulée par l'évolution démographique, les dynamiques et logiques internes au marché du travail, les fluctuations conjoncturelles, les

1. L'Organisme pour le Perfectionnement scolaire, la Transition et l'Insertion professionnelle (OPTI) et le Semestre de Motivation (SEMO) sont les deux structures de transition les plus mentionnées par les jeunes de notre échantillon. 
déséquilibres entre offre et demande selon les secteurs, ou encore la capacité de formation des entreprises ${ }^{2}$ (SEFRI, 2014).

Ce déséquilibre relatif entre offre et demande semble ainsi contribuer à transformer les pratiques à l'embauche qui, à leur tour, concourent à modifier le rapport entre offre et demande. Face à l'augmentation des candidatures, les employeurs tendent soit à privilégier les jeunes issus des filières scolaires les plus exigeantes, au détriment de ceux issus des filières dédiées à la poursuite d'études en apprentissage (Perriard, 2005), soit à préférer les candidats un peu plus âgés, gage à leurs yeux d'une plus grande maturité (Vanheerswyngehls, 1996).

La présente recherche qualitative est fondée sur 29 entretiens semi-directifs ${ }^{3}$ conduits dans le canton de Vaud avec 23 apprentis ou anciens apprentis et six recruteurs. Elle met à jour les pratiques et les attentes des recruteurs. En particulier, elle interroge les critères les plus usités par ces derniers pour apprécier si, par exemple, et comme l'a montré Vanheerswyngehls (1996), ils tendent à privilégier les candidats plus âgés, accentuant alors le rôle des structures de transition école-emploi et institutionnalisant leur présence.

Elle vise aussi à identifier le capital social dont disposent, ou non, les jeunes à la recherche d'une place d'apprentissage. Ces ressources ne constituent pas les seuls leviers d'action. Les stratégies mobilisées par les jeunes révèlent l'importance de ne pas se cantonner à suivre les voies prescrites dans les processus de recrutement. Elles suggèrent l'existence d'un curriculum caché - au sens de "la part des apprentissages qui n'apparaît pas programmée par l'institution scolaire" (Perrenoud, 1993, p. 61) - dans la recherche d'une place d'apprentissage mais qui néanmoins influence la probabilité de signer un contrat avec une entreprise formatrice. En d'autres termes, le curriculum caché consiste ici en la possession de savoirs, savoir-faire et notamment savoir-être qui, s'ils n'apparaissent pas explicitement au programme scolaire dispensé à l'école obligatoire, jouent pourtant un rôle actif dans les chances d'être retenu par une entreprise formatrice, participant ainsi à reproduire certaines inégalités sociales.

L'encadré 1 présente le fonctionnement du système scolaire vaudois, dans lequel s'enchâsse l'accès à l'apprentissage, la population et la méthode d'enquête. La première partie identifie les dimensions ordinaires du recrutement, tandis que la deuxième questionne les avantages potentiels que peut conférer un parcours scolaire atypique. La troisième partie met en évidence le rôle des stages préalables dans le processus de recrutement, révélant ainsi, au travers des critères de sélection, l'existence d'un curriculum caché, fondé en grande partie sur le «savoir-être » et les affinités entre individus. Une quatrième partie revient plus en détails sur la notion de feeling, apparue fréquemment dans les entretiens comme l'une

2. Ainsi, environ $40 \%$ des entreprises, en Suisse, ont la capacité de former des jeunes (SEFRI, 2014).

3. Ces entretiens ont tous été conduits par les étudiants et étudiantes du séminaire "Introduction à la sociologie de l'éducation » du semestre de printemps 2014, à l'université de Lausanne, que nous tenons à remercier pour la qualité de leur travail. 
des raisons invoquées à l'embauche. Enfin, une cinquième partie appréhende l'appréciation du rapport au temps comme indicateur d'autonomie. La conclusion contextualise cette injonction à l'autonomie (Ehrenberg, 2012) faite aux candidats à l'apprentissage, les renvoyant ainsi à leur propre responsabilité dans le succès ou l'échec de leur formation.

\section{Encadré 1 : Le système de formation dans le canton de Vaud : une orientation doublement précoce}

Les parcours de formation des jeunes qui constituent notre échantillon d'enquête se réfèrent aux différents espaces qui structurent la formation, tant obligatoire que post-obligatoire, dans le canton de Vaud, en Suisse.

Les interviewés ont suivi leur scolarité dans un système scolaire caractérisé par l'existence de trois filières de niveau dès le secondaire I $\left(^{*}\right)$ : la voie secondaire à options (VSO), la voie secondaire générale (VSG) et la voie secondaire baccalauréat (VSB). Ces filières regroupaient les élèves selon leurs capacités évaluées lors des deux années de cycle de transition, soit de 10 à 12 ans. Si la VSB, toujours existante, prépare les élèves à entrer au gymnase afin d'y obtenir le baccalauréat, la VSG devait les mener soit à des écoles de culture générale soit à entamer une formation professionnelle initiale. Enfin, la VSO était exclusivement prévue pour préparer les élèves les moins scolaires à entrer en formation professionnelle initiale.

Le panorama de la formation post-obligatoire de niveau secondaire II, soit après l'école obligatoire, est composé du gymnase où le baccalauréat est délivré, après trois ans d'études, aux élèves issus de la VSB. Des écoles de culture générale drainant principalement des élèves issus de la VSG délivrent, également au bout de trois ans, un Certificat de Culture Générale ; après une année de formation supplémentaire, il permet d'obtenir soit une Maturité professionnelle et un Certificat fédéral de capacité (CFC), soit une Maturité spécialisée ouvrant vers les Hautes écoles spécialisées (HES) et la Haute école pédagogique (HEP). Les deux-tiers des élèves s'orientent toutefois vers une formation professionnelle délivrant un CFC au bout de trois voire quatre ans. Il s'agit principalement des élèves de VSG et VSO, bien que, selon le type de métier et les exigences qui y sont liées, ce sont plutôt les uns ou les autres qui sont recrutés. Enfin, il existe des formations professionnelles délivrant, au bout de deux ans, une Attestation de formation professionnelle (AFP), et destinées aux élèves les moins scolaires. Le système scolaire vaudois se caractérise ainsi par une orientation doublement précoce des élèves vers une filière au secondaire (vers l'âge de 10-12 ans), puis, pour celles et ceux qui iront en formation professionnelle, le choix d'un métier dès l'âge de 14-15 ans.

${ }^{*}$ ) : en 2013, une nouvelle Loi sur l'Enseignement Obligatoire (LEO) a ramené le nombre de filières à deux.

\section{Obtenir une place d'apprentissage : quelques facteurs ordinaires}

Les facteurs ordinaires renvoient à des mécanismes précédemment identifiés dans les recherches scientifiques comme constitutifs des processus de construction des inégalités sociales dans le monde scolaire. Ils incluent en particulier la filière scolaire et son degré de prestige, mais également le capital social familial qui rend compte des 
ressources à disposition mobilisées par la famille pour faciliter l'accès de l'élève à une place d'apprentissage.

\subsection{Sortir d'une filière scolaire prestigieuse : un atout certain}

D’une manière générale, les résultats de la recherche corroborent d'autres études portant sur la transition école-emploi.

Tout d'abord, à l'instar de Haeberlin, Imdorf et Kronig (2004), la filière scolaire suivie semble influencer la qualité de la transition, notamment en termes de durée de recherche d'une place d'apprentissage, avec un temps de recherche d'autant plus long que les jeunes sont issus de filières scolaires peu prestigieuses.

De plus, si tous les jeunes issus de VSB, sans exception, ont trouvé une place d'apprentissage dans le domaine professionnel souhaité, ce n'est pas le cas d'une majorité de jeunes issus de VSO (3 sur 5), constat préalablement établi par Rastoldo (2006) dans le canton de Genève.

De fait, la responsabilité du recruteur ne se limite pas à la sélection d'un apprenti répondant aux critères de l'entreprise, mais intègre la dimension scolaire : le recrutement d'un apprenti ouvre à ce dernier l'accès à une formation en milieu scolaire (il s'agit des cours professionnels suivis un à deux jours par semaine en alternance avec la formation dans l'entreprise formatrice), dont les exigences peuvent être distinctes et supérieures à celles de l'entreprise. Le succès de l'apprentissage dépendant à la fois de la capacité à réussir scolairement, puisque les apprentis sont évalués en cours sur différentes connaissances, et dans l'intégration professionnelle, le recruteur est donc tenu d'évaluer simultanément ces deux dimensions.

\subsection{L'entourage familial, un précieux levier}

Les entretiens ont également révélé que le capital social (Bourdieu, 1980) des parents influence la probabilité de trouver rapidement une place, comme en témoigne cet extrait d'entretien avec un jeune ayant entamé un apprentissage de dessinateur en génie civil :

"Normalement, dans l'entreprise où je suis, ils prennent des apprentis tous les deux ans et je suis tombé juste dans l'année où ils ne cherchaient personne, mais j'ai eu un coup de pouce du patron que je connais très bien, que toute ma famille connaît et il a fait une proposition au directeur pour pouvoir me prendre en tant qu'apprenti parce qu'il voyait que j'étais motivé pour ce travail. » (Apprenti en $4^{\mathrm{ème}}$ année de dessinateur en génie civil, 19 ans)

Le capital social peut constituer une ressource importante sur laquelle s'appuient un quart des apprentis interviewés ; il est en effet une production collective à usage social correspondant à l'ensemble des ressources actuelles ou potentielles liées à la possession d'un réseau durable de relations plus ou moins institutionnalisées d'interconnaissance et d'interreconnaissance (Bourdieu, 1980) ; cependant, deux autres types de soutien familial interviennent (Monette \& Fournier, 2000). Les parents, et notamment la mère, ainsi que, dans une moindre mesure, les aînés de la fratrie, apportent également, dans bien des cas, un soutien 
émotionnel (encouragement, motivation) et instrumental (aide à la rédaction et relecture de $C V$ et lettres de motivation).

\section{Les parcours atypiques, un avantage pour les jeunes en quête d'apprentissage?}

Différentes études ont déjà montré que les parcours atypiques, au sens où ils ne s’inscrivent pas dans le modèle linéaire tel qu'envisagé par le système de formation suisse, sont loin de constituer des exceptions (Amos, 2007).

\section{Encadré 2 : Population d'enquête et méthodologie}

L'échantillon des jeunes interrogés $(n=23)$ est constitué de 11 hommes et 12 femmes âgés de 16 à 25 ans. Deux d'entre eux effectuaient leur 1 ère année d'apprentissage, six leur $2^{\text {ème }}$ année, quatre leur $3^{\text {ème }}$ année, un était en $4^{\text {ème }}$ année, sept avaient terminé. Cinq d'entre eux sont passés par des mesures transitoires. Les formations représentées concernent un agent d'exploitation (nettoyage et entretien des immeubles), une assistante dentaire, deux assistantes en pharmacie, un charpentier, deux coiffeuses, un dessinateur en génie civil, huit employés de commerce, deux gestionnaires de vente de détail, un installateur en chauffage, deux libraires, un pâtissier-confiseur et un polydesigner 3D (conception de décors pour magasins ou événements).

Les recruteurs ( $n=6$, quatre hommes et deux femmes) chargés du recrutement d'apprentis dans les entreprises, sont soit des chefs d'entreprise, soit des gérants de magasins ou encore des personnes rattachées spécifiquement à des services du personnel d'entreprises. Aucun des six n'occupait le même poste au sein de son entreprise. Toutes les personnes de notre enquête ont été approchées soit par le biais du réseau social de nos étudiants, soit en sollicitant leur accord directement sur leur lieu de travail.

Les entretiens ont été conduits de manière semi-directive et selon la méthode de l'entretien compréhensif (Kaufmann, 1996). Plus qu'une technique qui repose sur un processus itératif entre proximité et mise à distance, co-construction de l'information et production d'hypothèses, observations et interprétation des faits pour construire progressivement une théorie en partant d'une première exploration du terrain, l'entretien compréhensif participe également d'une conception de l'enquête en sciences sociales où l'enquêté n'a pas forcément raison, mais a « ses » raisons et « sa » logique qu'il s'agit d'identifier et de comprendre (Maulini, 2006).

Après avoir élaboré une première version de deux grilles d'entretien différentes, une pour les apprentis et l'autre pour les recruteurs, et avoir mené une première vague de 15 entretiens fin mars 2014, les grilles ont été réajustées en fonction des réponses obtenues. Si la grille concernant les apprentis a été partiellement modifiée pour approfondir la question des ressources dont les apprentis disposaient, celle relative aux recruteurs n'a quasiment subi aucune modification. A la suite de ces changements, une deuxième vague de 14 entretiens a été menée fin avril 2014, avec de nouveaux individus. 
Parmi les jeunes interviewés, cinq apprentis ont fréquenté une structure de transition et six ont préalablement étudié au Gymnase (quatre parmi eux l’ont terminé). La moitié de notre échantillon se caractérise ainsi par un parcours non linéaire.

Si tous les parcours atypiques ne sont pas valorisés par les recruteurs, certains semblent être appréciés sinon recherchés.

Dans le cas de l'entrée en apprentissage, un parcours non linéaire, pour autant que la période de transition ait été active (avoir fait d'autres études, un séjour linguistique à l'étranger, avoir commencé un premier apprentissage puis avoir bifurqué ou encore être passé par une mesure de transition) confere, souvent, un avantage certain : celui d'être plus âgé, synonyme aux yeux des recruteurs de davantage de "maturité ». Parmi les jeunes interviewés, ceux âgés de 17 ans et plus et qui sont restés actifs pendant leur transition ont trouvé une place en l'espace de deux mois.

A l'inverse, ceux âgés de 15 ou 16 ans ont connu une durée de recherche plus longue, aucun n'ayant trouvé une place en moins de trois mois, mais plus souvent en quatre à six mois. Dans ce contexte, passer par une mesure de transition peut donc s'avérer un avantage, comme en témoignent les extraits suivants :

"Un jeune qui sort de VSO, ben je suis un peu réticente, mais un VSO qui a fait une année d'OPTI (Organisme pour le Perfectionnement scolaire, la Transition et l'Insertion professionnelle) avec de bons résultats à l'OPTI, ben il y a aucun souci parce qu'il a acquis une année de maturité en plus. Moi je pense vraiment que tout se joue sur la maturité. " (Responsable RH, femme, entreprise d'assurances)

"L'OPTI m'a aussi aidée. Au niveau du dossier de candidature, on faisait même des entretiens d'appréciation pour voir comment on se débrouillait. C'est comme des faux entretiens et après on te propose des améliorations, de te tenir plus correctement, ou des trucs comme ça. " (Apprentie en $3^{\text {ème }}$ année d'employée de commerce, 20 ans)

Par ailleurs, avoir poursuivi d'autres études post-obligatoires au préalable confère un avantage important. Parmi les six jeunes ayant étudié au Gymnase avant de se lancer à la recherche d'une place d'apprentissage, cinq ont trouvé en moins de deux mois (un seul a cherché une place pendant trois mois avant de trouver). Pour les candidats à l'apprentissage n'ayant pas étudié au Gymnase, huit sur treize, soit la majorité, ont cherché une place pendant plus de quatre mois (quatre d'entre eux ont cherché pendant six mois ou plus).

A titre d'exemple, un apprenti en conception de projet de chauffage ayant étudié quelques années au Gymnase sans achever sa formation, a spécifié que l'entreprise dans laquelle il avait postulé ne lui avait rien demandé hormis son $C V$ et ses notes du Gymnase. Un simple entretien a suffit à son embauche :

"Je n'ai pas fait de stage. Même si la plupart des apprentis que je connais doivent faire un stage avant de commencer (...) Faut dire que ça donnait déjà assez confiance le fait que j’avais fait 
quand même les premières années du lycée (...) Donc, on m'a fait signer le contrat de travail. Je crois que je suis un cas un peu particulier parce que tous les apprentis que je connais ont dî faire au moins une semaine de stage. " (Ancien apprenti en conception de projet de chauffage, 25 ans)

Ces profils semblent donc être, comme en témoigne l'extrait suivant, particulièrement recherchés par certains types d'employeurs et dans certains domaines d'activités et probablement d'autant plus dans les PME où les apprentis sont susceptibles d'être plus rapidement soumis aux impératifs de rendement :

"J'estime que dans le type d'activité que l'on a, une forme de maturité, à savoir... on n'est pas forcément plus mature à 18 ans qu'à 16 ans mais enfin, on estime que les gens de plus de 18 ans ont déjà une certaine maturité et souvent ont déjà un cursus scolaire plus avancé" (Recruteur et formateur d'apprentis, homme, entreprise d'ébénisterie)

Ces extraits, et particulièrement le dernier, permettent également de mettre en évidence une inadéquation entre les attentes de certains employeurs face aux jeunes qu'ils recrutent en apprentissage et leur degré de maturité et de formation en fin de scolarité obligatoire. Tout se passe comme si certaines places d'apprentissage, participant pourtant d'un système de formation professionnelle pensé pour intégrer les jeunes immédiatement à la sortie de l'école obligatoire, ne leur étaient pas directement destinées, augmentant alors le nombre de transitions indirectes et donc les coûts de maintien dans les structures transitoires.

\section{Le stage en entreprise ou la consécration 3 des critères de sélection informels}

"Y'en a de plus en plus qui ne comprennent pas l'importance de leur stage" (Apprenti en $3^{\text {ème }}$ année d'employé de commerce, 22 ans).

Les stages en entreprise effectués par les jeunes constituent certainement l'étape la plus importante dans le processus d'obtention d'une place d'apprentissage. Les entretiens avec les recruteurs permettent de distinguer deux types de stages remplissant chacun des rôles bien spécifiques.

Le premier, que nous nommerons le stage d'orientation, intervient alors que les jeunes sont encore à l'école obligatoire, souvent entre l'avant-dernière et la dernière année scolaire (lorsqu'ils ont entre 14 et 16 ans). Ces stages, effectués soit pendant les vacances scolaires soit pendant les heures d'école, offrent la possibilité de passer quelques jours au sein d'une entreprise afin de découvrir " la réalité du métier ». Ils contribuent donc à aider les jeunes à préciser leurs choix de formation. Si les entreprises formatrices semblent souvent disposées à leur offrir cette possibilité, cela questionne les différents rôles qu'elles sont amenées à jouer au sein de la formation professionnelle initiale. En 
effet, un recruteur a déclaré se refuser à proposer des stages exploratoires, pointant les difficultés temporelles et économiques qu'ils généraient et estimant que cette tâche relevait davantage des services d'orientation que de sa responsabilité.

Lors du processus de recrutement intervient une deuxième forme de stage. Il s'agit du stage de sélection. Les entreprises convoquent des jeunes dont les dossiers ont été préalablement retenus. A une exception près, la totalité des jeunes et des recruteurs de notre enquête ont évoqué cette étape liminaire à l'obtention de la place comme une condition fondamentale sans laquelle aucun contrat ne se signe.

De manière factuelle, les potentiels futurs apprentis sont amenés à évoluer, sur une courte période d'environ trois jours à une semaine, au sein de l'entreprise, sous le regard attentif d'un ou plusieurs collaborateurs. Un certain nombre d'éléments sont alors évalués in situ. Pour les employeurs, l'évaluation des connaissances n'est pas l'enjeu majeur de cette procédure, puisqu'elles ont déjà été évaluées par le biais du dossier scolaire et/ou de différents tests, qu'ils soient internes ou externes à l'entreprise. Selon les recruteurs de notre échantillon, ce sont les savoir-faire, comme savoir témoigner d'une capacité à agir de manière rapide et adéquate à des situations professionnelles, et surtout le savoir-être des jeunes, qui sont appréciés. Savoir-être qui devient alors, aux côtés des savoirs et des savoir-faire, une composante de la connaissance (Kergoat, 2007) :

"Tout au long du recrutement, c'est... on essaie beaucoup de voir... sill a des compétences aussi de savoir-être (...) Le savoir-être, c'est vis-à-vis des autres apprentis surtout, c'est là qu'on voit beaucoup. Les jeunes qui causent, qui causent, qui causent, qui racontent plein de choses qui ne sont pas adaptées ou alors les jeunes qui sont renfermés, qui ne se lient pas, qui ne montrent pas d'intérêt. » (Recruteur, femme, administration publique)

Sans toujours évoquer frontalement la notion de "savoir-être ", devenue, selon Ségal (2005), un mot tabou dans le langage des ressources humaines, les recruteurs interrogés ont tous maintes fois mentionné l'importance qu'ils accordent aux attitudes et aux comportements tant lors des entretiens que lors des stages. Précisons que dans le contexte de l'entreprise, la notion de "savoir-être " renvoie alors avant tout à une prescription de normes comportementales apparaissant, selon Lichtenberger (1997, in Ségal, 2006), sous différents registres, comme le comportement, les compétences relationnelles, méthodologiques, transverses, génériques et la prise de responsabilité.

Ainsi, pouvoir répondre à ces critères, variables selon le type de monde en vigueur au sein des différentes entreprises (Boltanski et Thévenot, 1991), semble bien, comme l'ont déjà évoqué Perret-Clermont et Zittoun (2002), influer sur les chances de réussite à l'embauche et donc plus largement sur la transition entre l'école et l'emploi. A ce titre, l'étude d'Imdorf et Seiterle (2015) portant sur les difficultés d'insertion des jeunes d'origine étrangère sur le marché de l'apprentissage, en Suisse, a mis en évidence les obstacles sérieux que ces jeunes rencontraient à l'embauche, particulièrement au sein du monde domestique de 
l'entreprise, organisé autour d'un principe de familiarité dont dépend la cohésion sociale. Les propos de certains recruteurs illustrent clairement le poids de certaines composantes du savoir-être durant le stage :

"Alors le jeune, on va tout de suite voir s'il est renfermé, s’il a une certaine appétence, une certaine ouverture et dans son langage, on va tout de suite voir, enfin percevoir en tout cas l'éducation et le respect. Il y a des choses très simples. Alors il y a le parler, il y a le visuel qui joue aussi énormément." (Gérant de magasin, homme, grande distribution)

Outre certaines capacités relationnelles, un certain type de langage ainsi que le respect de certains codes esthétiques sont également attendus. A cet égard, certains jeunes ont témoigné d'une conscience face à ces critères constitutifs du curriculum caché et du rôle des parents dans l'acquisition de ces compétences.

"Mon père m'a vachement drillé, comment je devais me comporter dans les stages. Fallait montrer que tu sais travailler. J'ai un père qui est assez comme ça. Il m'a vraiment expliqué comment il fallait se tenir, des trucs bêtes! Mais, par exemple, comment serrer la main, faire une bonne poigne de main, des choses comme ça. " (Apprenti en $3^{\text {ème }}$ année de polydesigner 3D, 18 ans)

L'extrait suivant s'avère encore plus explicite sur l'importance du savoir-être. En effet, le formateur estime ici nécessaire de faire un "travail culturel " sur l'apprenti, soit de passer par une étape d'acculturation professionnelle pour laquelle un type d'habitus préalable, ou des "prédispositions ", comme les nomme le formateur, s'avèrent selon lui nécessaires :

"Il y a tout un travail culturel pour que cette personne puisse un jour être envoyée de façon individuelle chez un client, parce que c'est quelque chose que je fais très volontiers si la personne a des prédispositions et une attitude positive par rapport à, en tout cas celles que j'attends de sa part, c'est que je puisse envoyer quelqu'un assez rapidement seul chez un client pour prendre des mesures, pour des petites actions, prendre une série de photos par exemple. " (Recruteur et formateur d'apprentis, homme, entreprise d'ébénisterie)

Ces pratiques de sélection basées sur la possession d'un savoir-être particulier s'avèrent ainsi discriminantes car elles pénalisent les jeunes qui ne possèdent pas les prédispositions requises, prédispositions découlant davantage du milieu social d'origine et donc de la socialisation primaire que de la socialisation secondaire qui s'opère dans le cadre de l'école obligatoire. Selon Kergoat (Op. cit), deux raisons pourraient expliquer l'importance accordée au savoir-être lors du recrutement. La première résiderait dans une vision naturalisée où : "à l'inverse des savoirs et des savoir-faire, les savoir-être ne seraient que peu modifiables, améliorables par le biais de la formation" (p. 18) tandis que la seconde découlerait du fait "que les candidats - postulant à une formation et non à un emploi n'auraient pas encore acquis de compétences professionnelles entendues comme savoir et savoir-faire" (p. 18), rendant alors l'évaluation de ces derniers relativement difficile. 


\section{Avoir le «feeling»: les affinités avant tout?}

En plus des savoir-faire et savoir-être évalués lors des stages et des entretiens, un autre critère à l'embauche s'est révélé particulièrement significatif. Evoqué tant par les jeunes que par les recruteurs de notre corpus, le feeling s'avère un critère de sélection tout aussi central qu'informalisable pour les recruteurs. Cet anglicisme renvoie, dans le discours de nos interviewés, à une impression positive laissée par l'autre, à la reconnaissance d'une "éthique de travail» commune et compatible (Maeder \& Nadai, 2009), à l'expression d'affinités électives et sélectives jouant très directement, comme en témoignent les passages suivants :

"Je crois qu'au fond, le critère principal c'est que le courant passe, que l'on s'imagine... parce que l'on va tout de même passer trois ans avec cette personne, dans une relation tout de même assez proche, avec des exigences et une responsabilité sur cette personne. " (Recruteur, femme, responsable d'un office cantonal vaudois)

"Il y a des candidats qu'on sent tout de suite bien. Ils ont amené directement un dossier complet avec un Basic-check ${ }^{4}$ qui nous a bien convenu. Les monteurs, tout de suite, ils arrivent "Ah lui, il est super!". Quand je cause avec eux, je sens tout de suite la chose. Et bien on n'attend pas. On fait une proposition de contrat tout de suite pour ne pas laisser passer un bon élément." (Recruteur, homme, entreprise d'électricité)

Du côté des jeunes, la présence d'un bon feeling avec les futurs employeurs et collaborateurs est tout aussi importante. Dans de nombreux cas, c'est le signal que l'entretien s'est bien passé et que leurs chances d'obtenir la place sont élevées. Interrogé sur le déroulement de l'entretien, un interviewé répond ainsi :

"Ouais, il y avait un très bon feeling. Donc moi je suis parti de l'entretien et jétais là, ouais, c'est bon quoi! C'est bon, j'ai la place!" (Apprenti en $3^{\text {ème }}$ année d'employé de commerce, 22 ans)

Placé à l'ombre des critères formels de sélection, comme les résultats scolaires, le feeling ressenti par le recruteur constitue un critère à l'embauche tout à fait significatif. Ceci d'autant plus quand il s'agit de petites entreprises formatrices où, comme l'ont relevé Bentabet et Théry (2005), les recruteurs sont principalement à la recherche de qualités comportementales, faisant du jugement spontané, de l'intuition et du " coup de cœur " des justifications suffisantes à l'intégration dans l'entreprise.

4. Le Basic-check est un test payant fourni par une société privée. Mesurant les compétences dans différents domaines comme l'arithmétique, la géométrie ou encore les langues, il a pour objectif d'informer les futurs employeurs des capacités des candidats à satisfaire aux exigences de la profession souhaitée. 


\section{Le rapport au temps: un indicateur d'autonomie}

En plus d'intégrer les savoir-être, le curriculum caché comprend également un rapport au temps spécifique et la capacité à donner et à rendre compte du sens de son projet d'apprentissage.

La confrontation des pratiques des recruteurs et des expériences des apprentis saisit un processus de recrutement et de recherche d'apprentissage qui s'inscrit dans la durée et peut schématiquement être dépeint ainsi :

Figure 1. Schéma du processus de recrutement et de recherche d'apprentissage

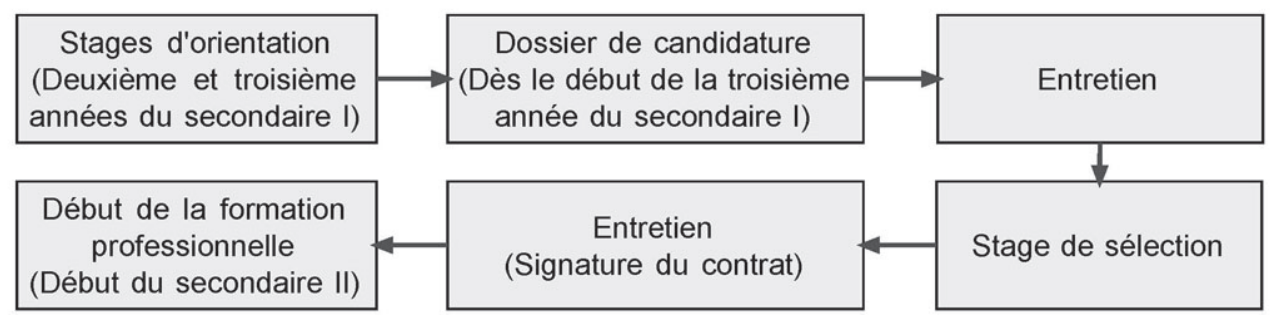

Source : Auteurs.

Pour les futurs apprentis, la construction du processus débute dès la deuxième année de cycle secondaire I, avec un stage obligatoire d'une semaine qu'ils doivent organiser. C'est l'occasion d'une première réflexion sur le type de profession et d'environnement de travail envisagé, mais aussi de premiers contacts professionnels qui peuvent ensuite nourrir le capital social de l'élève, les réseaux qu'il pourra mobiliser par la suite. Pour les recruteurs, la temporalité du recrutement varie selon la taille et l'organisation de l'entreprise, mais peut durer près d'une année.

Cette temporalité longue, et la capacité de l'élève à s'y inscrire, produit une deuxième différenciation, notamment parmi les élèves aux trajectoires linéaires : anticiper, construire son projet par le biais des stages constituera ensuite un outil pour convaincre un recruteur potentiel :

"Parfois, il y a des gens qui demandent longtemps, longtemps à l'avance. Parfois même un, voire deux ans à l'avance. Il m'est arrivé d'avoir des gens qui ont demandé bien bien bien à l'avance. Et ben, ils ont souvent eu raison. Dans la mesure où ça a permis de faire des entretiens, des stages de longue haleine et de confirmer que c'était effectivement des gens extrêmement motivés. " (Entretien, Recruteur, homme, entreprise d'ébénisterie) 
La capacité d'anticipation est donc perçue par les recruteurs comme garante de la motivation du futur apprenti, tout comme la capacité à se singulariser dans le processus de recrutement :

"J'anime souvent des ateliers dans le cadre du salon des métiers (...) tout d'abord, on fait une conférence sur comment chercher une place d'apprentissage, puis après on fait des simulations d'entretien où les jeunes peuvent venir une demi-heure nous présenter leur dossier, etc. Et puis, là aussi, on distribue nos cartes, comme ça, et puis là aussi on reçoit quelques dossiers. Donc, c'est beaucoup du bouche à oreille aussi (...) Alors il y a des jeunes où ça aboutit sur un contrat après ouais, alors ça c'est sûr. Quand il y a un chouette feeling qui est passé, ben le jeune qui a déjà pris le temps de venir au salon des métiers, c’est qu'il s’intéresse, s’il a pris le temps... Bon moi, en général, j'anime le dimanche donc voilà, qui vient le dimanche aprèsmidi faire un entretien, c'est qu'il a de l'intérêt donc euh, ouais, alors ça peut déboucher sur des contrats à la fin quoi. » (Responsable RH, femme, entreprise d'assurances)

Implicites à ce rapport à la temporalité, deux dimensions semblent structurer le recrutement, et témoigner d'une attente spécifique des recruteurs. Ainsi, interrogée sur les "bons critères " de sélection des apprentis, une recruteuse répond : "Ces deux critères, je pense que c'est vraiment autonomie et motivation. " L'expression de cette motivation est attendue dans le processus de recrutement.

In fine, capacité d'anticipation et d'action apparaissent, aux yeux des recruteurs, corrélées avec le degré d'autonomie et de motivation du candidat à l'apprentissage. Faire preuve de persévérance et d'autodiscipline semble témoigner, auprès du recruteur, d'une "éthique de travail" commune (Maeder \& Nadai, 2009) et jouer ainsi favorablement sur les chances d'être recruté. La capacité à obtenir une place d'apprentissage dépend ainsi, au-delà des caractéristiques scolaires de l'élève, de sa capacité à se présenter non seulement comme capable de fonctionner de façon stratégique, d'opérer des choix rationnels, mais également de subjectiver ses choix et d'être apte à se construire une action autonome et une identité propre (Dubet, 1994).

\section{Conclusion}

L'obtention d'une place d'apprentissage, qui plus est dans le domaine professionnel souhaité, est l'aboutissement d'un processus complexe qu'il convient d'analyser à l'aune des dynamiques spécifiques au marché de l'apprentissage.

La situation de concurrence caractérisant les places d'apprentissages les plus prisées et la nécessité, pour certains employeurs, d'embaucher des apprentis rapidement opérationnels, conduisent à valoriser, lors du recrutement, un certain type de ressources scolaires et sociales, de savoir-faire et de savoir-être.

A ce titre, la prise en compte du milieu social d'origine des enquêtés aurait permis d'affiner cet axe d'analyse en y reliant plus précisément ces critères de sélection. Nous 
y avons renoncé en raison du nombre trop restreint d'observations permettant de conduire une analyse heuristique.

Néanmoins, nous avons observé que le soutien familial aux différentes étapes de la recherche d'une place d'apprentissage était un élément important, que ce soit dans la constitution du dossier de candidature, en termes de soutien émotionnel, ou encore par la mobilisation des réseaux sociaux.

Réfléchir à partir du curriculum caché (rappelons qu'il s'agit de la possession de savoirs, savoir-faire et notamment savoir-être qui, s'ils n'apparaissent pas explicitement au programme scolaire dispensé à l'école obligatoire, jouent pourtant un rôle actif dans les chances d'être retenu par une entreprise formatrice, participant ainsi à reproduire certaines inégalités sociales), conduit à caractériser l'apprenti idéal, du point de vue des recruteurs ; cette caractérisation débouche sur un critère d'âge, les recruteurs recherchant des jeunes plus âgés plutôt que ceux dont la trajectoire scolaire est linéaire et qui sortent juste de l'école obligatoire ; cela conduit à préférer les apprentis les plus dotés en capital scolaire et ceux passés par les mesures de transition.

Principalement évalué par les futurs employeurs lors des stages et des entretiens, ce curriculum caché comporte la maîtrise d'un certain type de savoir-être, variable selon le monde valorisé par l'entreprise qui recrute et son éthique de travail (Imdorf \& Seiterle, 2015 ; Maeder \& Madai, 2009), voire selon celle du recruteur en charge du recrutement. Le poids du feeling, évoqué tant par les recruteurs que par les apprentis dans le processus de recrutement, témoigne de l'importance de l'identification, chez le candidat, d'un certain nombre de dispositions communes.

De même, autonomie, réflexivité, capacité à témoigner de sa motivation et sens pratique apparaissent comme des composantes centrales de ce curriculum caché. Comme le relève Ehrenberg (2012, p. 13), l'injonction à l'autonomie, en tant que "capacité à saffirmer de manière maîtrisée et appropriée, devient un ingrédient essentiel de la socialisation à tous les niveaux de la hiérarchie sociale ". L'affirmation de soi devient alors à la fois une norme, parce qu' elle est contraignante, et une valeur, parce qu'elle est désirable (Ehrenberg, ibid.). Ainsi, l'injonction à l'autonomie faite aux candidats à l'apprentissage dans la procédure de recrutement ne constitue donc pas une originalité dans le contexte des sociétés occidentales contemporaines mais, à l'inverse, une règle implicite qui tend à faire reposer le poids de la responsabilité de la réussite ou de l'échec sur les seules épaules des individus. 


\section{Bibliographie}

Amos J. (2007), «Transition école-emploi : apports de la recherche TREE », in Behrens M. (Dir.), La transition de l'école à la vie active ou le constat d'une problématique majeure Neuchâtel, IRDP, pp. 43-54.

Bentabet E. \& Théry M. (2005), Les organismes paritaires collecteurs agréés, acteurs du changement des comportements de formation des petites entreprises, Marseille, Relief $\mathrm{n}^{\circ} 11$, Céreq.

Boltanski L. \& Thévenot L. (1991), De la justification. Les économies de la grandeur, Paris, Gallimard.

Bourdieu P. (1980), "Le capital social ", Actes de la recherche en sciences sociales, vol. 31, pp. 2-3.

Commission européenne (2010), Europe 2020 - Une stratégie pour une croissance intelligente, durable et inclusive, Strasbourg.

Cortesi S. \& Imdorf C. (2013), « Le certificat fédéral de capacité en Suisse - Quelles significations sociales pour un diplôme hétérogène ? ", Cahiers de la recherche sur l'éducation et les savoirs, Hors Série n ${ }^{\circ} 4$, pp. 91-108.

DFJC (2014), Mesures de transition T1 dans le canton de Vaud. Consulté le 7 septembre 2014 depuis le site internet: http://www.vd.ch/fileadmin/user_upload/themes/formation/ orientation/fichiers_pdf/filieres_infos/resume_mesures_T1_vd.pdf

Dubet F. (1994), Sociologie de l'Expérience, Seuil, Paris.

Ehrenberg A. (2012), La Société du malaise, Paris, Odile Jacob.

Eurostat (2014), Taux de chômage par sexe, âge et nationalité (\%). Consulté le 20 Septembre 2014 depuis le site internet : http://appsso.eurostat.ec.europa.eu/nui/submitViewTableAction.do;jsessionid=9ea7d07d30d776ccf54e65034e9fb161 ae049aa0356d. e34MbxeSaxaSc40LbNiMbxeNbxiOe0

Fibbi R. Kaya B. \& Piguet E. (2003), Nomen est omen: Quand s'appeler Pierre, Afrim ou Mehmet fait la différence. Synthèse du rapport mené dans le cadre du PNR 43 (FNS), Berne, Direction du programme PNR 43-FNRS.

Haeberlin U., Imdorf C. \& Kronig W. (2004), Chancenungleichheit bei der Lehrstellensuche: Der Einfluss von Schule, Herkunft und Geschlecht, Berne/Aarau, Centre suisse de coordination pour la recherche en éducation.

Häfeli K. \& Schellenberg C. (2009), Les facteurs de réussite dans la formation professionnelle des jeunes à risque, Rapport, Bienne, CDIP.

Imdorf C. \& Seiterle N. (2015), « La formation professionnelle dans le cadre des réseaux d'entreprises formatrices comme aide à l'intégration des jeunes issus de l'immigration ", 
in Felouzis G. et Goastellec G., (Eds), Les inégalités scolaires en Suisse. Ecole, société et politiques éducatives, Genève, Peter Lang, pp. 141-160.

Kaufmann J.-C. (1996), L'entretien compréhensif, Paris, Nathan.

Kergoat P. (2007), «Une redéfinition des politiques de formation. Le cas de l'apprentissage dans les grandes entreprises ", Formation Emploi, n 99, pp. 13-28.

Lamamra N. (2011), « La formation professionnelle en alternance, un lieu de transmission des normes de genre ?", PTO, vol. 17, n 4, pp. 330-345.

Lamamra (2014), « Le bon corps au bon endroit. Incorporation des normes de genre en formation professionnelle en alternance " in Collet I. \& Dayer C. (Eds), Raisons éducative. Former envers et contre le genre.

Lamamra N. \& Masdonati J. (2009), Arrêter une formation professionnelle : mots et maux d'apprenti-e-s. Lausanne, Antipodes.

LINK (2013), Baromètre des places d'apprentissage. Août 2013, Rapport condensé, Berne, SEFRI.

Maeder C. \& Nadai E (2009), "The Promises of Labor : The Practices of Activating Unemployment Policies in Switzerland", in Marci Giugni (Dir.), The Politics of Unemployment in Europe. Policy Responses and Collective Action, Farnham/Burlington, Ashgate, pp. 67-81.

Maulini (2006), " Note de lecture sur l'ouvrage de Kaufmann J.-C. (1996) », L'entretien compréhensif, Paris, Nathan. Consulté le 8/02/2016 : http://www.unige.ch/fapse/SSE/ teachers/maulini/2006/sem-rech-note-lecture.pdf

Monette M. \& Fournier G. (2000), "Soutien social et adaptation à la transition entre les études et le marché du travail. ", in Fournier G. et Monette M. (dir.), L'insertion socioprofessionnelle : un jeu de stratégie ou un jeu de hasard?, Québec, Les Presses de l'Université de Laval, pp. 57-76.

OFS (2014), Taux de chômage des jeunes au sens du BIT. Consulté le 12 Novembre 2014 depuis le site internet : http://www.bfs.admin.ch/bfs/portal/fr/index/themen/00/10/blank/ ind44.indicator.30000303.4406.html

Perret-Clérmont A.L. \& Zittoun T. (2002), "Esquisse d'une psychologie de la transition ", Education permanente, Revue suisse pour l'éducation des adultes, 36(1), pp. 12-15.

Perrenoud P. (1993), "Curriculum : le formel, le réel, le caché ", in Houssaye J. (dir.), La pédagogie : une encyclopédie pour tous aujourd'hui, Paris, ESF, pp. 61-76.

Perriard V. (2005), Transition de l'école obligatoire vers la formation professionnelle : les facteurs explicatifs des difficultés actuelles, Rapport de l'URSP, Lausanne, URSP. 
Rastoldo F. (2006), "Les élèves de 9e s'expriment sur leur choix d'orientation ", Panorama, Vol. 1, pp. 12-20.

Ségal E. (2005), Les compétences " relationnelles » en question, université d'Evry Vald'Essonne, Centre Pierre Naville.

Ségal E. (2006), "Les “savoir-être”, un angle mort des débats sur la compétence », L’Homme et la société, n $164 / 4$, pp. 97-113.

SCRIS (2011), Orientation à l'issue de la scolarité obligatoire et des filières de transition. Résultats de l'enquête "choix 2010 ", Lausanne, SCRIS.

SEFRI (2013), Un marché des places d'apprentissages dynamique. Consulté le 8 Septembre 2014 depuis le site internet : http://www.sbfi.admin.ch/aktuell/medien/00483/00594/index. html?lang=fr\&msg-id $=50803$

SEFRI (2014), La formation professionnelle en Suisse. Faits et données chiffrées, Rapport, Berne, SEFRI.

Sigerist P. (2003), "Formation professionnelle : le chemin de la promotion, Panorama, $\mathrm{n}^{\circ}$ 6, pp. 20-23.

Valli S. (2012), Transition de l'école obligatoire à la formation professionnelle: problématique et état des lieux, Rapport établi à la demande de la Direction de l'enfance, la jeunesse et la cohésion sociale (DEJCS) de la ville de Lausanne, Lausanne, DEJCS.

Vanheerswynghels A. (1996), Prolongation de la scolarité : 10 ans après. Enquêtes longitudinales sur le devenir scolaire et professionnel de jeunes ayant fréquenté l'enseignement secondaire technique et professionnel et se trouvant en situation de retard scolaire important, Rapport de recherche effectué à la demande de la ministre de l'Education de la Communauté française, Bruxelles, CSER. 